\title{
ZBTB46 is a shear-sensitive transcription factor inhibiting endothelial cell proliferation via gene expression regulation of cell cycle proteins
}

\author{
Yu Wang ${ }^{1} \cdot$ He-Ying Sun ${ }^{1} \cdot$ Sandeep $\operatorname{Kumar}^{2} \cdot$ Maria del Mar Puerta ${ }^{1} \cdot$ Hanjoong Jo $\mathbb{(}^{2} \cdot$ Amir Rezvan (1)
}

Received: 1 September 2017 / Revised: 8 February 2018 / Accepted: 20 February 2018 / Published online: 8 June 2018

(c) The Author(s) 2018. This article is published with open access

\begin{abstract}
ZBTB46 is a transcription factor identified in classical dendritic cells and keeps dendritic cells in a quiescent state. Chromatin immunoprecipitation sequencing in dendritic cells has identified over 1300 potential gene targets of ZBTB46, affecting many processes including cell cycle. Endothelial cells (ECs) also express ZBTB46 and are mostly in a quiescent non-proliferative state. While EC proliferation is a critical process in development, dysregulation of EC proliferation as seen in areas of disturbed flow play an important role in many disease processes such as atherosclerosis, pulmonary hypertension, transplant vasculopathy, neointimal hyperplasia, and in-stent restenosis. We studied the role of ZBTB46 in ECs, hypothesizing that it inhibits EC proliferation. Using a model of disturbed flow in mice, we found that ZBTB46 is expressed in murine arterial ECs in vivo, and is downregulated by disturbed flow. In vitro results using HAECs showed that cell confluence and laminar shear stress, both known physiological conditions promoting EC quiescence, led to upregulation of ZBTB46 expression. Adenoviral-mediated overexpression of ZBTB46 in vitro caused reduced EC proliferation, and increased number of cells in the $\mathrm{G}_{0} / \mathrm{G}_{1}$ phase of cell cycle, without affecting apoptosis or senescence, while siRNA knockdown of ZBTB46 negated the known inhibitory role of unidirectional laminar shear stress on EC proliferation. ZBTB46 overexpression also led to a broad suppression of genes involved in cell cycle progression including multiple cyclins and cyclin-dependent kinases, but an increase in the CDK inhibitor CDKN1A. Phosphorylation of the retinoblastoma protein was also decreased as assessed by Western blot. Tube formation on Matrigel was reduced, suggesting an inhibitory role for ZBTB46 in angiogenesis. Further research is required to investigate the potential role of ZBTB46 in specific pathologic conditions and whether it can be targeted in a therapeutic manner.
\end{abstract}

These authors contributed equally: Yu Wang, He-Ying Sun.

Electronic supplementary material The online version of this article (https://doi.org/10.1038/s41374-018-0060-5) contains supplementary material, which is available to authorized users.

Amir Rezvan

amir.rezvan@emory.edu

1 Department of Medicine, Division of Cardiology, Emory University School of Medicine, Atlanta, GA, USA

2 Department of Medicine, Division of Cardiology, Wallace H. Coulter Department of Biomedical Engineering, Georgia Institute of Technology and Emory University School of Medicine, Atlanta, GA, USA

\section{Introduction}

ZBTB46 (also known as BTBD4, zDC, BZEL, RINZF, and ZNF340) is a transcription factor belonging to the BTB-ZF (broad complex, tramtrack, bric-à-brac, and zinc finger) family of transcription repressors, and considered a marker of classical dendritic cells (cDCs) within the immune system $[1,2]$. ZBTB46 is expressed in quiescent cDCs, and downregulated in response to TLR4 activation. Chromatin immunoprecipitation sequencing (ChIP-Seq) in cDCs has identified over 1300 gene targets, affecting many processes including cell cycle [3]. While ZBTB46 expression was also noted in endothelial cells (ECs) of the splenic vasculature, its role in ECs has not been studied [1]. As most ECs in the adult mouse vasculature are quiescent at baseline, we hypothesized that similar to its role in $\mathrm{CDC}$ quiescence, ZBTB46 contributes to the quiescent state of ECs and its downregulation is required for EC proliferation. It is well 
established that arterial ECs exposed to a disturbed flow pattern (exhibiting a low and oscillatory shear stress profile), such as inner curvatures or bifurcations become activated and have a higher turnover rate [4-7]. Shear stress is also known to affect EC gene expression including important transcription factors regulating EC proliferation such as NFkB, AP1, EGR1, KLF2, and KLF4 [8-14], and regulates the cell cycle through regulation of cell cycle proteins such as CDKN1A (p21 $1^{\text {cip1 }}$ ) and phosphorylation of retinoblastoma protein $(\mathrm{Rb})[15,16]$. Therefore, in this study we also investigated whether ZBTB46 is regulated by shear stress, and whether ZBTB46 expression levels affect EC proliferation in response to shear stress. While EC proliferation is a critical process in development, and a significant disruption of normal EC proliferation is not compatible with life in organisms with complex vasculature such as humans or mice, more subtle dysregulations of EC proliferation as seen in areas of disturbed flow play an important role in many disease processes such as atherosclerosis, pulmonary hypertension, transplant vasculopathy, neointimal hyperplasia, and in-stent restenosis [17].

\section{Materials and methods}

\section{Partial carotid ligation surgery}

Animal studies were performed using $\mathrm{C} 57 \mathrm{Bl} / 6 \mathrm{~J}$ mice (8-10-weeks-old male, Jackson Laboratory, stock \#000664) according to the approved Institutional Animal Care and Use Committee protocol by Emory University. Mice left common carotid arteries were partially ligated under anesthesia, and development of low and oscillatory shear stress was determined by ultrasound measurements as previously described $[18,19]$. Briefly, three of four caudal branches of the left common carotid artery (external carotid, internal carotid, and occipital artery) were ligated with 6-0 silk suture, and the superior thyroid artery was left intact. Mice were euthanized 2 days post ligation and carotid arteries were dissected and removed for analysis.

\section{Intimal RNA isolation from carotid arteries}

Total RNA from intima was separately obtained from the left common carotid artery (LCA) and right common carotid artery (RCA) at 2 days post ligation, using the flushing technique as previously described $[18,19]$.

\section{Isolation of endothelial RNA from lesser curvature (LC) and greater curvature (GC) of mouse aortic arch}

Aortic arch was harvested from mice and opened en-face. The endothelium was placed against a nitrocellulose membrane soaked in isopropanol for 5 min, the media and adventitia were peeled away leaving the intima adherent to the nitrocellulose membrane, and RNA was extracted using Qiagen miREasy kit as previously described [20].

\section{Cell culture and shear stress experiments}

Human aortic endothelial cells (HAECs) were obtained commercially (GIBCO, C0065C or PromoCell, C-12271) and cultured according to the manufacturer's standard protocol. HAECs were seeded at various density to meet the purposes of different studies, and maintained in EC medium containing growth supplements (EBM-2, Lonza, CC-3516, CC-4176 or PromoCell, C-22011) with $2 \%$ FBS. HAECs between passage 6 and 7 were used for experiments. For shear experiments, confluent HAECs in $100 \mathrm{~mm}$ dishes were exposed to steady laminar shear (LS, $15 \mathrm{dyn} / \mathrm{cm}^{2}$ ), or oscillatory shear (OS, $\pm 5 \mathrm{dyn} / \mathrm{cm}^{2}$ ) conditions for 2 days using a cone-and-plate shear device as previously described [20,21]. For shear experiments with gain of function or loss of function components, the commercially available Ibidi pump system (Ibidi, Germany) was used to reduce reagent use, and set up according to manufacturer instructions. LS $\left(15 \mathrm{dyn} / \mathrm{cm}^{2}\right)$ or OS $\left( \pm 5 \mathrm{dyn} / \mathrm{cm}^{2}\right)$ was applied for 2 days to $80 \%$ confluent HAECs cultured overnight on microchannel slides ( $\mu$-Slide $\mathrm{I}^{0.4}$ Luer, Ibidi, Germany) coated with $40 \mu \mathrm{g} / \mathrm{ml}$ collagen (Collagen Type I, BD Biosciences 35-4236).

\section{Measurement of cell counts}

HAECs were seeded in multiple wells at same subconfluent density $\left(2.0 \times 10^{\wedge} 4 /\right.$ well in 24 -well plates $)$. Transduced cells were seeded $24 \mathrm{~h}$ after infection alongside non-treated control cells. Cells were counted manually using a hemocytometer every $24 \mathrm{~h}$ for 4 days.

\section{Adenoviral-mediated overexpression of ZBTB46}

HAECs were cultured in $25 \mathrm{~cm}^{2}$ cell culture flasks (Corning) to $70 \%$ confluent. Adenoviral infection of HAECs were carried out by replacing $6 \mathrm{ml}$ culture medium with $3 \mathrm{ml}$ medium containing control Ad-CMV-GFP (Vector Biolabs, Malvern, PA, 1060) or Ad-CMV-GFP-h-ZBTB46 (Vector Biolabs, Malvern, PA, ADV-228076) at an adenoviral particle to cell ratio of 20:1. After $4 \mathrm{~h}$ incubation, adenovirus-containing medium were replaced by normal endothelial medium without antibiotics and cells were cultured overnight before subculture for experimental usage. For ZBTB46 localization study, HAECs were infected with adenovirus-expressing FLAG-tagged (N-terminal) full-length human ZBTB46 (CMV promoter, Ad-FLAG-h-ZBTB46, Vector Biolabs, Malvern, PA) or 
FLAG-tagged (N-terminal) truncated version of human ZBTB46 (amino acid 420-498 deleted) lacking the zinc finger domains and a predicted nucleus localization sequence (CMV promoter, Ad-FLAG-h-ZBTB46-d420498aa, Vector Biolabs, Malvern, PA). The adenoviral infection process for FLAG-tagged ZBTB46 constructs are the same as the GFP constructs described above, except that we used a dose of 20:1 for Ad-FLAG-h-ZBTB46 and 5:1 for Ad-FLAG-h-ZBTB46-d420-498aa to get similar ZBTB46 expression levels in HAECs transduced with these two different constructs due to different transduction efficiency.

\section{siRNA-mediated downregulation of ZBTB46}

HAECs were cultured to $80 \%$ confluence and transfected with $100 \mu \mathrm{M}$ control siRNA (Cat. D-001810-10-05, GE Healthcare Dharmacon Inc.) or ZBTB46 siRNA (Cat. L325, Thermo Sic) using oligofectamine (Cat.12252-011, Invitrogen) per manufacturer recommendations. Cells were cultured for $24 \mathrm{~h}$ before being transferred to Ibidi slides for shear stress experiments.

\section{Immunofluorescence staining for Ki67 and FLAG}

For Ki67 immunofluorescence staining, culture media were removed from cells and replaced with PBS for a brief wash. HAECs were then fixed for $15 \mathrm{~min}$ at room temperature (RT) with $10 \%$ formalin and washed twice with PBS. Fixed cells were permeabilized with $0.1 \%$ Triton X-100 for 15 min at RT. After blocking with $20 \%$ goat serum for $30 \mathrm{~min}$ at RT, cells were incubated overnight with rabbit anti-Ki67 hAb (Abcam, Cambridge, MA, ab15580, $2 \mu \mathrm{g} / \mathrm{ml}$ in 5\% goat serum), washed three times with PBS, and incubated for $2 \mathrm{~h}$ at RT protected from light with Alexa-fluor 568-labeled goat anti rabbit IgG (1:500 dilution). For FLAG immunofluorescence staining, culture media were removed from cells and replaced with PBS for a brief wash. HAECs were then fixed for $15 \mathrm{~min}$ at RT with $4 \%$ paraformaldehyde and $4 \%$ sucrose and washed twice with PBS. Fixed cells were permeabilized with $0.25 \%$ Triton X-100 for $5 \mathrm{~min}$ at RT. After blocking with $10 \%$ bovine serum albumin for 30 min at RT, cells were incubated overnight with monoclonal Anti-FLAG M2 (Sigma-Aldrich, F1804, St. Louis, MO. 1:1000 dilution), washed three times with PBS, and stained for $2 \mathrm{~h}$ at RT protected from light with Dylight 548-labeled goat anti mouse IgG (1:1000 dilution). Following incubation with secondary antibodies, cells were washed three times with PBS, and counter stained with DAPI (Sigma-Aldrich, D-9542, St. Louis, MO) for $15 \mathrm{~min}$ at RT and washed with PBS. Micrographs were taken using a fluorescent microscope.
Flow cytometry analysis of cell cycle assay

Cells were trypsinized and detached from culture flasks, washed twice with wash buffer (PBS $+0.1 \%$ BSA) and re-suspended at $1-2 \times 10^{6} \mathrm{cell} / \mathrm{ml} .1 \mathrm{ml}$ aliquots of cells were fixed by adding 3 $\mathrm{ml}$ cold $\left(-20^{\circ} \mathrm{C}\right)$ absolute ethanol dropwise while gently agitating and stored overnight at $-20^{\circ} \mathrm{C}$ prior to staining. For propidium iodide (PI) staining, fixed cells were washed twice and pelleted with centrifugation. $1 \mathrm{ml}$ of PI (Life Technologies, Eugene, OR) staining solution ( $3.8 \mathrm{mM}$ sodium citrate, $40 \mu \mathrm{g} / \mathrm{ml}$ PI) and $0.5 \mu \mathrm{g}$ of RNase A were added to cell pellet and mixed well and incubated $3 \mathrm{~h}$ at $4{ }^{\circ} \mathrm{C}$ prior to flow cytometry analysis. Flow cytometry was performed using a BD LSR II flow cytometer and data were analyzed using FlowJo software.

\section{Cell apoptosis assay}

Cell apoptosis assay was carried out by using a PE Annexin $\mathrm{V}$ apoptosis detection kit with 7-AAD (BioLegend, San Diego, CA) and following manufacture's instruction with minor modifications. Briefly, cells were washed twice with cold BioLegend's Cell staining buffer and re-suspended in Annexin $\mathrm{V}$ binding buffer at a concentration of $0.5 \times 10^{6}$ cells $/ \mathrm{ml}$. $50 \mu \mathrm{l}$ of cell suspension was transferred into a $5 \mathrm{ml}$ test tube, and $2.5 \mu \mathrm{l}$ of PE Annexin V and $2.5 \mu \mathrm{l}$ of 7-AAD viability staining solution were added. After incubation for $15 \mathrm{~min}$ at RT in the dark, $300 \mu \mathrm{l}$ of Annexin V binding buffer was added to each tube. Specimens were analyzed by flow cytometry with proper settings.

\section{Senescence-associated beta-galactosidase (SA- $\beta$-gal) staining}

To study cell senescence, in situ SA- $\beta$-gal staining was performed according to manufacturer protocol. Briefly, cells were grown in 12-well cell culture plates, washed with PBS, and fixed with $2 \%$ formaldehyde with $0.2 \%$ glutaraldehyde in PBS for 5 min at RT. Cells were washed twice with PBS and incubated with $\beta$-gal staining solution $\left(150 \mathrm{mM} \mathrm{NaCl}, 2 \mathrm{mM} \mathrm{MgCl}_{2}, 5\right.$ $\mathrm{mM}$ potassium ferricyanide, $5 \mathrm{mM}$ potassium ferrocyanide, 40 $\mathrm{mM}$ citric acid/sodium phosphate buffer, $\mathrm{pH}$ 6.0), containing 1 $\mathrm{mg} / \mathrm{ml}$ x-gal (Abcam, Cambridge, MA, ab144388) dissolved in DMF overnight at $37^{\circ} \mathrm{C}$. Thereafter, cells were washed with PBS and methanol and allowed to air dry. Blue stain was visualized and pictures were taken by bright field to visualize blue staining, and phase contrast microscopy to count total cells using an Olympus IX71 microscope.

\section{Tube formation assay}

Tube formation assays were carried out as described previously with minor modifications [22, 23]. HAECs were evenly seeded at a density of $2 \times 10^{4}$ cells/well in 48 -well cell culture plates pre- 
coated with Matrigel (Corning, 354234) and cultured overnight. After overnight incubation, micrographs were taken at $2 \times$ magnification using an Olympus IX71 microscope. Tube formation (total tube length per well and network branch points) was quantified using ImageJ.

\section{RNA extraction and quantitative PCR}

Total RNA of animal tissue samples and cultured cell samples were isolated using the Direct-zol RNA MiniPrep kit (Zymo Research, Irvine, CA). RNA concentrations were then determined using a BioTek Synergy H1 microplate reader (BioTek Instruments, Winooski, VT); $200 \mathrm{ng}$ of RNA was used for reverse transcription to make cDNA using a High Capacity cDNA Reverse Transcription Kit (Applied Biosystems by Thermo Fisher Scientific). Quantitative PCR was carried out on a LightCycler instrument (Roche Applied Science, Indianapolis, IN) in glass capillaries, using PlatinumTaq DNA polymerase (Invitrogen) and SYBR green (Invitrogen) dye. Estimation of amplified gene products were normalized to $18 \mathrm{~s}$ or GAPDH. Human primer sequences used were as follows: ZBTB46: forward 5'-CGGGAAGAAGTTCACGCGG-3', reverse 5'-CTGCA CACCTTGCACACATAC-3'; 18s: forward 5'-AGGAATT GACGGAAGGGCACCA-3', reverse 5'-GTGCAGCCCC GGACATCTAAG-3'; GAPDH: forward 5'-GGATTTGG TCGTATTGGG-3', reverse 5'-GGAAGATGGTGATGGG ATT-3'; CDK1: forward 5'-TTTTCAGAGCTTTGGGCA CT-3', reverse 5'-CCATTTTGCCAGAAATTCGT-3'; CD K2: forward 5'-ACTGAGGGTGTGCCCAGTA-3', reverse 5'-GAGGGGAAGAGGAATGCCAG-3'; CDK4: forward 5'-ACCCGTGGTTGTTACACTCTG-3', reverse 5'-ACAG AAGAGAGGCTTTCGACG-3'; CDK6: forward 5'-CTG GCCTAGAGATGTTGCCC-3', reverse 5'-GTGAGACAG GGCACTGTAGG-3'; CCNA2: forward 5'-TGCTGGAG CTGCCTTTCATTT-3', reverse 5'-ATGCTGTGGTGCTT TGAGGTA-3'; CCNB1: forward 5'-CGGGAAGTCACT GGAAACAT-3', reverse 5'-AAACATGGCAGTGACAC CAA-3'; CCND1: forward 5'-TCCTCTCCAAAATGCCAGAG-3', reverse 5'-GGCGGATTGG AAATGAACTT3'; CCNE1: forward 5'-TCCAGGAAGAGGAAGGC AAAC-3', reverse 5'-TTGTCAGGTGTGGGGATCAG-3'; Rb1: forward 5'-AGTGCTGAAGGAAGCAACCC-3', reverse 5'-TGTCCACCAAGGTCCTGAGA-3'; CDKN1A: forward 5'-CTGCCCAAGCTCTACCTTCC-3', reverse 5'ATCTGTCATGCTGGTCTGCC-3'; CDKN1B: forward 5'TCTGAGGACACGCATTTGGT-3', reverse 5'-GCATT TGGGGAACCGTCTGA-3'.

\section{Western blot}

Samples were harvested in phosphate-buffered RIPA lysis buffer (Boston BioProducts, Ashland, MA). After incubation on ice for $30 \mathrm{~min}$, samples were centrifuged at $12,000 \mathrm{rpm}$ for $10 \mathrm{~min}$ and the supernatants were collected. Total protein concentrations were determined by the Pierce BCA protein Assay (Thermo Scientific, Rockford, IL). Protein lysates were boiled and separated on $10 \%$ SDS-PAGE gels and transferred to PVDF membranes. Following blocking, membranes were incubated with primary antibodies (diluted accordingly in 5\% BAS/ TBST). Primary antibodies and dilutions used were rabbit anti-ZBTB46 (Novus, NBP1-88506, 1:500), mouse anti$\mathrm{Rb}$ (Cell Signaling 9309, 1:1000), rabbit anti-phospho (Ser797)-Rb (Cell Signaling 9301, 1:1000), mouse anti- $\beta$ actin (Sigma-Aldrich, A5316, 1:10,000), and rabbit antiGAPDH (Santa Cruz, sc-25778, 1:10,000). After overnight incubation with the primary antibody at $4{ }^{\circ} \mathrm{C}$, membranes were washed and incubated with secondary antibodies (anti-mouse IgG-HRP (Santa Cruz, sc-2005, 1:10,000), anti-rabbit IgG-HRP (Santa Cruz, sc-2004, $1: 10,000)$ ), diluted in 5\% BSA/TBST for $1 \mathrm{~h}$ at RT. Bands were developed by adding Immobilon Western chemiluminescent HRP substrate (Millipore, Billerica, MA) to the membranes and captured using Kodak image station 4000 $\mathrm{mm}$ Pro. Protein content was quantified using ImageJ and normalized to GAPDH or $\beta$-actin.

\section{Statistics}

All studies were performed in duplicates or triplicates with at least three or more replicates per assay. Data are presented as mean \pm SEM. Statistical significance was assessed using ANOVA and Bonferroni's multiple comparisons test by GraphPad Prism software. In some cases, a Student's $t$ test was used to assess significance between groups when no other variables were considered. A value of $P<0.05$ was considered statistically significant.

\section{Results}

\section{ZBTB46 is expressed in arterial ECs in vivo and is downregulated in areas exposed to disturbed flow}

In a study using GFP-expressing ZBTB46 transgenic mice, Satpathy et al. noted that splenic vasculature ECs expressed ZBTB46 [1]. We first examined whether healthy quiescent arterial EC express ZBTB46 in vivo, using RNA obtained from the mouse common carotid artery. It is well established that ECs exposed to a disturbed flow pattern, such as inner curvatures or bifurcations, become activated and have a higher turnover rate [4-7]. To be able to compare the baseline ZBTB46 expression levels in quiescent ECs with activated ECs, we used an established model of acutely induced disturbed flow in mice by partial 

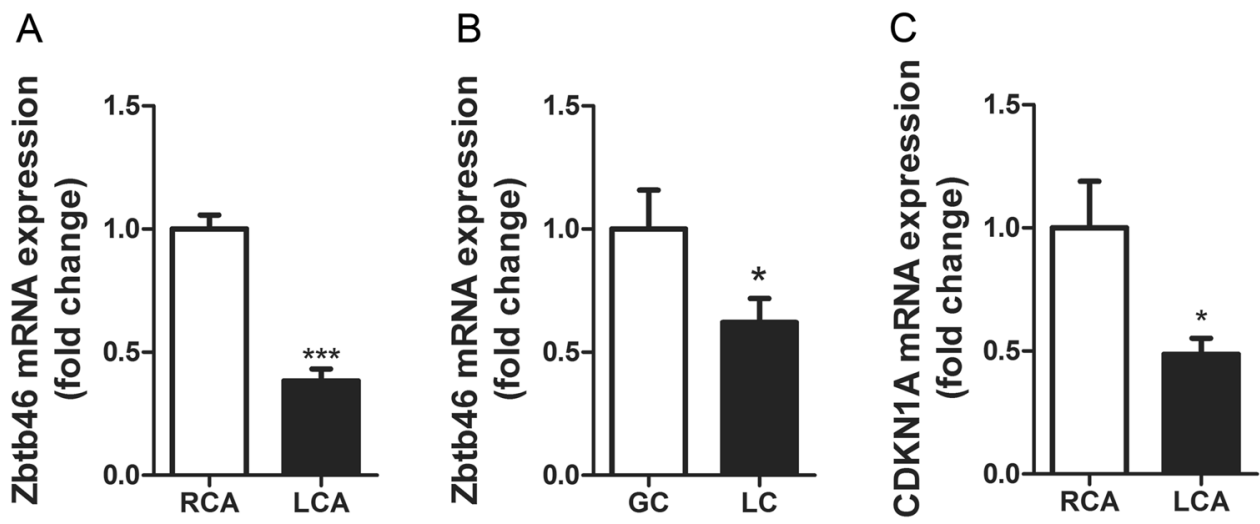

Fig. 1 ZBTB46 mRNA is expressed in mouse arterial endothelial cells in vivo and is downregulated in areas exposed to disturbed flow where EC proliferation is increased, and correlates with CDKN1A (p21) gene expression. We performed a partial ligation procedure in $\mathrm{C} 57 \mathrm{Bl} / 6$ mice which produces a disturbed flow profile in the LCA inducing endothelial cell activation, while retaining a unidirectional physiologic flow in the RCA where ECs are quiescent. EC-enriched RNA were isolated 2 days post ligation from the LCA and RCA, and ZBTB46 and CDKN1A (p21) mRNA expression in the LCA were compared to the RCA as control. ZBTB46 mRNA expression was also compared in the

ligation of the left common carotid artery (LCA), which exposes the ECs to low shear stress, and flow reversal during diastole, in the LCA, while ECs in the right common carotid artery (RCA) remain exposed to unidirectional laminar shear stress of physiologic amplitude $[18,19]$. Two days following the partial ligation procedure in C57Bl/6 mice, EC-enriched intimal RNA (Supplemental Fig. 1) was collected from the LCA and RCA, and ZBTB46 mRNA expression was assessed using qPCR. Our results show that ZBTB46 is expressed at baseline in samples collected from the RCA, and is significantly downregulated in the LCA (exposed to disturbed flow) by over $50 \%$ (Fig. 1a). To confirm this finding, we used a second model comparing the effect of more chronic differences in flow patterns on ZBTB46 mRNA expression, comparing gene expression by qPCR of intimal RNA isolated from the greater curvature (GC) of the aortic arch with the lesser curvature (LC) as previously described [20]. ECs in the GC are exposed to stable unidirectional flow, while ECs in the LC are chronically exposed to disturbed flow [6]. Consistent with the partial ligation model, we observed a similar pattern of ZBTB46 expression being downregulated in the LC where EC are chronically exposed to disturbed flow (Fig. 1b). These results show for the first time that ZBTB46 is expressed in arterial ECs and is downregulated in response to disturbed flow in vivo. We also verified the known effect of disturbed flow on EC proliferation (using en-face staining of Ki67 as a marker of cell proliferation) [24] in the partial carotid ligation model greater curvature (GC) vs. the lesser curvature (LC) of the aortic arch. a, c Endothelial cell ZBTB46 and CDKN1A(p21) mRNA expression in right common carotid artery (RCA) vs. left common carotid artery (LCA, partially ligated) showing downregulation of ZBTB46 and CDKN1A in the LCA. $* P<0.05, * * * P<0.01$ two-tailed $t$-test, $n=9$. b ZBTB46 mRNA expression in the greater curvature (GC) vs. the lesser curvature (LC) of the aortic arch. Intimal RNA was isolated from the LC and GC of the aortic arch in C57B1/6 mice and ZBTB46 mRNA expression in the LC was compared to the GC showing downregulation of ZBTB46 in LC. $* P<0.05$ one-tailed $t$-test, $n=7$

in vivo, and found that, as expected, EC proliferation is significantly increased in the partially ligated LCA compared to RCA control 2 days post ligation (Supplemental Fig. 2). Shear stress has been shown to affect the expression of the cyclin-dependent kinase inhibitor CDKN1A (p21 ${ }^{\text {Cip1 }}$ ) in EC, involved in shear stress regulation of EC proliferation $[15,16]$. We therefore examined the gene expression of CDKN1A in EC-enriched mRNA from the partially ligated LCA 2 days post ligation compared to the non-ligated RCA as control. Our results showed that CDKN1A was significantly reduced in the partially ligated artery (Fig. 1c).

\section{ZBTB46 is expressed in quiescent ECs in vitro, and is downregulated in proliferating cells and cells exposed to OS stress}

To assess the role of ZBTB46 on EC proliferation, we studied the effect of two known conditions affecting EC proliferation, i.e. contact inhibition and unidirectional laminar shear stress, on ZBTB46 expression in cultured HAECs. To understand the expression pattern of ZBTB46 in proliferative vs. contact inhibited ECs, we measured ZBTB46 mRNA level by qPCR in HAECs at various culture densities and post confluence. Interestingly, our results show that ZBTB46 mRNA expression is equally low in all groups of proliferating non-confluent cells; however, its expression is markedly increased (up to 5-fold) upon confluence and sustained thereafter (Fig. 2a). Correspondingly, robust increase of ZBTB46 protein was also evident by 
A

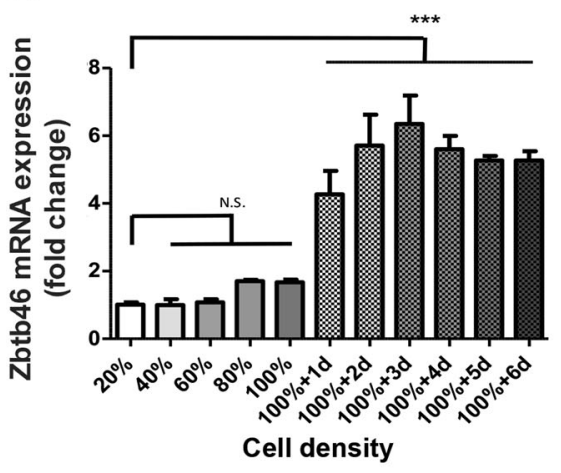

D

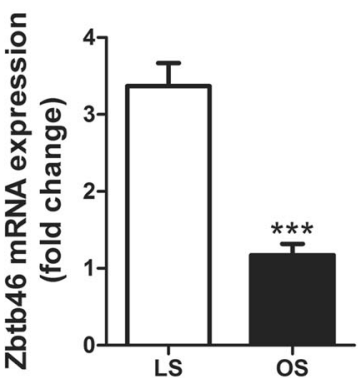

Fig. 2 Expression of ZBTB46 in endotheal cells at both mRNA and protein levels is induced in confluent human aortic cells in vitro and is shear sensitive, being significantly downregulated by low and oscillatory shear stress. a ZBTB46 mRNA expression in HAECs cultured at various densities $(20-100 \%)$ and up to 6 days post confluence. $* * * P<0.001$, compared to the $20 \%$ confluent group, one-way ANOVA, $n=6$. b, c Western blot assay for ZBTB46 protein content in $50 \%$ confluent HAECs compared to HAECs cultured 2 days

Western blot in cells cultured for 2 days post confluence compared to cells at $50 \%$ confluence (Fig. $2 \mathrm{~b}$, c). We then studied ZBTB46 expression in ECs subjected to different shear stress patterns in vitro using a cone-and-plate shear system. We used unidirectional laminar shear stress of 15 $\mathrm{dyn} / \mathrm{cm}^{2}(\mathrm{LS})$ as a representation of physiologic shear stress and compared to oscillatory shear stress of $\pm 5 \mathrm{dyn} / \mathrm{cm}^{2}$ (OS) representing the shear stress in areas exposed to disturbed flow for 2 days [20, 21]. HAECs exposed to OS conditions showed significantly decreased ZBTB46 mRNA expression (Fig. 2d) and protein content (Fig. 2e, b, f) compared to LS. These data demonstrate that EC expression of ZBTB46 is regulated by cell confluence and shear stress, and is downregulated in proliferating cells.

\section{ZBTB46 overexpression suppresses EC proliferation without inducing cell death}

We found that non-proliferating ECs express higher level of ZBTB46 compared to proliferative ECs; however, it is unclear whether ZBTB46 expression level has a causative

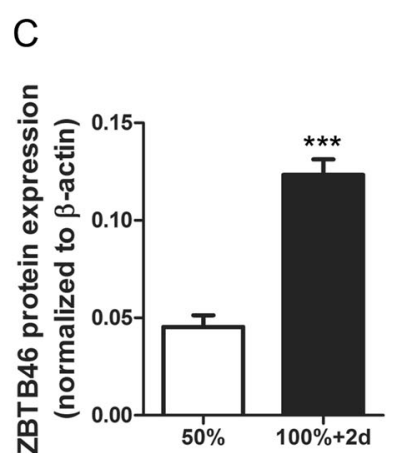

$\mathrm{F}$

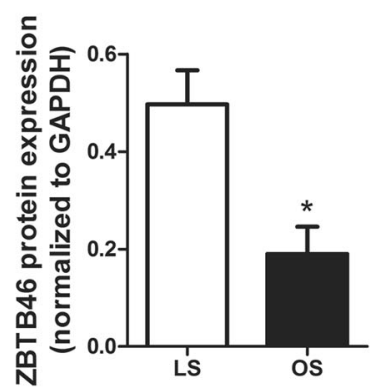

post confluent, quantified using ImageJ. $* * * P<0.001$, two-tailed $t$ test, $n=4$. d-f HAECs were cultured under unidirectional laminar shear stress (LS, $15 \mathrm{dyn} / \mathrm{cm}^{2}$ ) or low and oscillatory shear stress (OS, $\pm 5 \mathrm{dyn} / \mathrm{cm}^{2}$ ) for 2 days using a cone-and-plate in vitro shear system. $\mathbf{d}$ ZBTB46 mRNA expression by qPCR. e, f ZBTB46 protein expression by Western blot, quantified using ImageJ. $* P<0.05$, $* * * P<0.001$, $n=6$

role in reduced EC proliferation. Here we tested the hypothesis that high ZBTB46 levels suppress EC proliferation. We used adenoviral transduction of ZBTB46 to overexpress ZBTB46 in HAECs. HAECs were infected with adenovirus co-expressing GFP and human ZBTB46 transcripts or control adenovirus (GFP only) at an adenoviral particle to cell ratio of 20:1, alongside a baseline no treatment control (NTC). Robust increase in both ZBTB46 mRNA and protein levels were observed in ZBTB46transduced cells compared to controls (Fig. 3a-c). To examine the effect of ZBTB46 on cell proliferation, ZBTB46-overexpressing HAECs (24 h post infection) and control cells were sub-cultured at low density and cultured for 4 days, and live cells were counted manually by hemocytometer (with Trypan Blue exclusion of dead cells) every $24 \mathrm{~h}$. The proliferation curves clearly demonstrate that ZBTB46 overexpression resulted in marked reduction in live cell numbers at day 1 and any given time point thereafter compared with control groups (Fig. 3d), with no significant effect on cell death as measured by manual counting of Trypan Blue-positive cells at day 2 (Fig. 3e). 

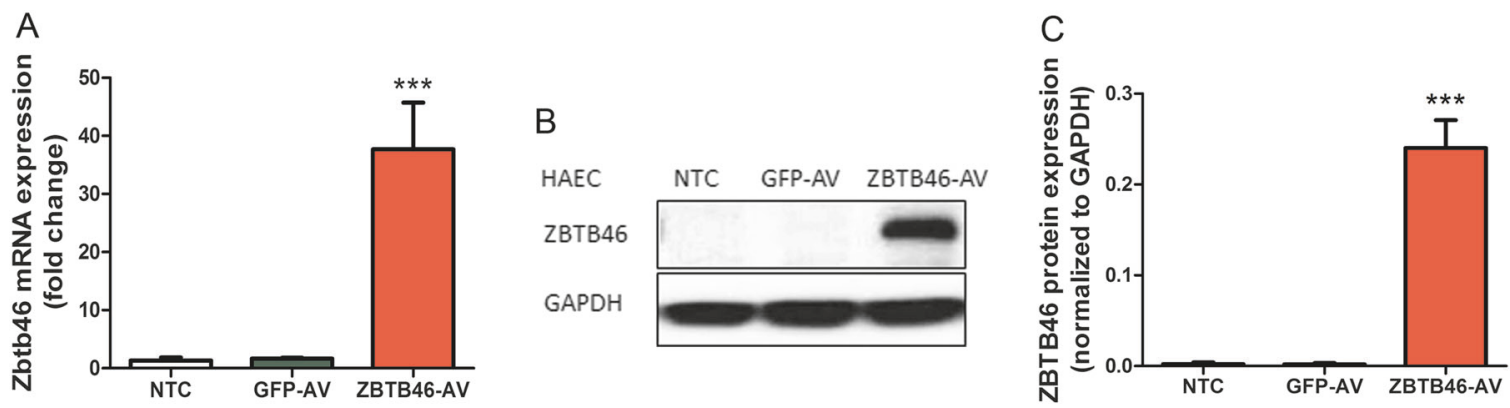

D

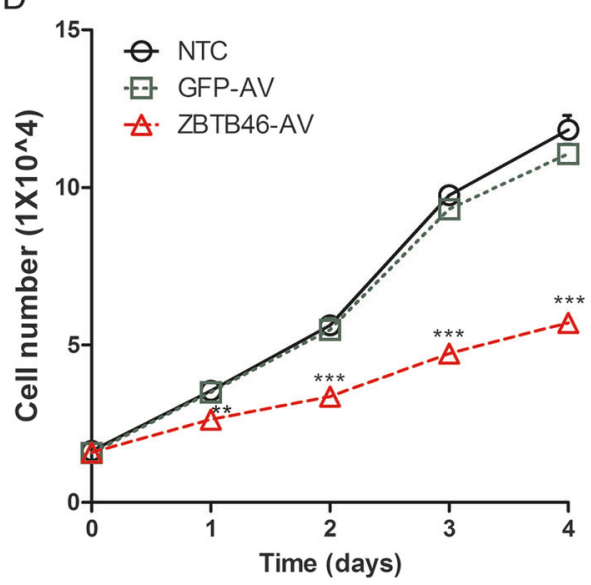

Fig. 3 Adenoviral-mediated overexpression of ZBTB46 results in a significant increase in ZBTB46 mRNA and protein expression in HAECs, leading to significant supression of EC proliferation. HAECs were transfected with adenovirus transducing both ZBTB46 and GFP as a marker for transduction efficiency (ZBTB46-AV) or adenovirus transducing only GFP as control (GFP-AV) at an adenoviral particle to cell ratio of 20:1, and cultured alongside non-treated control (NTC) HAECs. a-c ZBTB46 mRNA measured by qPCR and protein level

\section{ZBTB46 overexpression affects cell cycle, without affecting baseline apoptosis and senescence in HAECS}

Given the above results and that a ChIP-Seq assay in cDCs revealed multiple cell cycle genes as potential targets for ZBTB46 [3], we assessed the role of ZBTB46 on cell cycle in ECs using flow cytometry analysis of cell cycle by propidium iodide (PI) DNA staining in HAECs cultured in vitro (Fig. 4a). Our data revealed that ZBTB46 overexpression resulted in significantly more cells in the $G_{0} / G_{1}$ phase and fewer cells in the $S$ phase (Fig. 4b). We then sought to assess whether ZBTB46 overexpression increased apoptotic live cells. Cells were double stained with 7-AAD (live-dead marker) and Annexin V (apoptosis marker) to assess the effect of ZBTB46 overexpression on cell apoptosis. Flow cytometry results exhibited no significant difference in frequency of Annexin V-positive cells (as percentage of live transduced cells, gated on GFPpositive, 7-AAD-negative cells), suggesting that ZBTB46 overexpression did not lead to increased apoptosis (Fig. 4c). To distinguish non-proliferating cells from proliferating cells, we performed immunofluorescent staining with Ki67 antibody as a marker of proliferating cells [24] in ZBTB46-overexpressing and control cells. ZBTB46transduced cells showed significantly less Ki67-positive staining compared to GFP controls (Fig. 4d, e), confirming an increased ratio of non-proliferating cells. The decreased number of Ki67-positive cells in addition to the cell cycle analysis results suggest an increase in the number of ECs exiting the cell cycle $\left(\mathrm{G}_{0}\right)$. Cells in $\mathrm{G}_{0}$ are considered to be quiescent; however, this population of cells also includes senescent cells. In order to distinguish between quiescent and senescent cells, we assessed the effect of ZBTB46 on cell senescence, using a SA- $\beta$-gal staining assay which results in a positive staining of senescent cells. ZBTB46overexpressing cells did not show a significant increase in senescent cells (Fig. 4f), suggesting that the ZBTB46induced increase in $\mathrm{G}_{0}$ cells is due to increased number of quiescent but not senescent cells. 
A

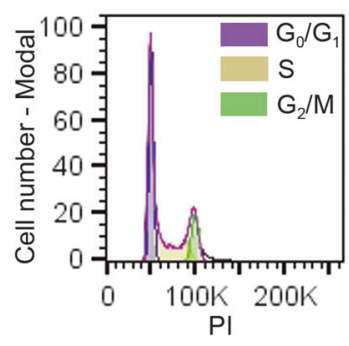

$\mathrm{B}$

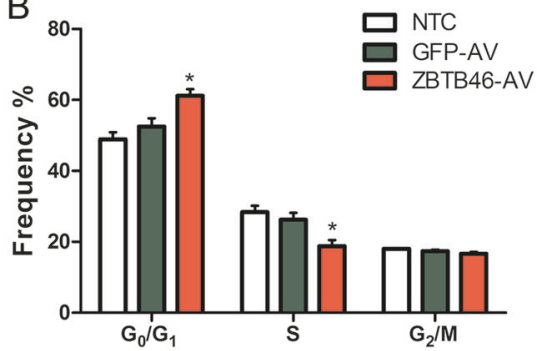

C

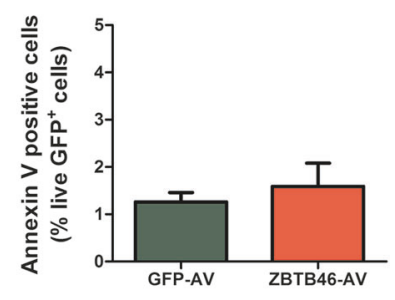

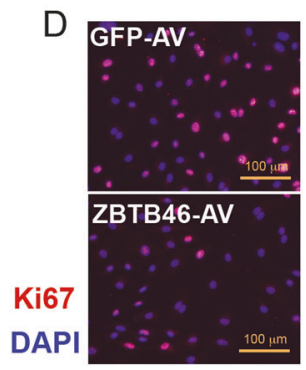

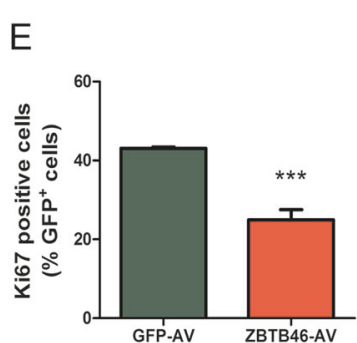

Fig. 4 Overexpression of ZBTB46 leads to higher percentage of cells in the $G_{0} / G_{1}$ phase, lower percentage of cells in the $S$ phase, and lower number of Ki67-positive cells, but no significant change in apoptosis or senescence. HAECs were infected with adenovirus transducing ZBTB46 or GFP control. Transduced HAECs were seeded at simliar sub-confluent density $24 \mathrm{~h}$ after infection, alongside non-treated cells as baseline control and assayed after 2 days. a Representative flow cytometry histogram of cell populations assigned to different cell cycle phases $\left(\mathrm{G}_{0} / \mathrm{G}_{1}, \mathrm{~S}\right.$, and $\left.\mathrm{G}_{2} / \mathrm{M}\right)$ based on intensity of PI staining (reflecting DNA content), gated on single cell population. b Flow cytometry quantification of cell numbers ( $\%$ of total cells) in each cell cycle phase. $* P<0.05$, compared to GFP control group, one-way ANOVA, $n=4-6 /$ group. c Frequency of Annexin V-positive cells

\section{ZBTB46 expression affects shear stress regulation of EC proliferation}

In order to assess the effect of ZBTB46 on shear stress regulation of EC proliferation, we used loss of function and gain of function experiments in HAECs exposed to laminar shear stress or oscillatory shear stress, respectively, using the Ibidi shear stress system in vitro followed by Ki67 staining to measure cell proliferation. ZBTB46 was downregulated in HAECs using siRNA to ZBTB46 (and compared to control non-targeting siRNA) in order to assess the role of ZBTB46 downregulation under the proliferation inhibited LS condition, and in a second set of experiments ZBTB46 was overexpressed using transduced HAECs infected with ZBTB46-AV (compared to control-AV infection) to assess the role of ZBTB46 overexpression under the proliferative OS condition. Following treatment with either siRNA or AV, HAECs were exposed to LS (15 $\left.\mathrm{dyn} / \mathrm{cm}^{2}\right)$ or OS $\left( \pm 5 \mathrm{dyn} / \mathrm{cm}^{2}\right)$ for 2 days using the commercially available Ibidi shear stress system. LS treatment of HAECs led to about a 50\% decrease in EC proliferation when compared to cells exposed to OS as expected.

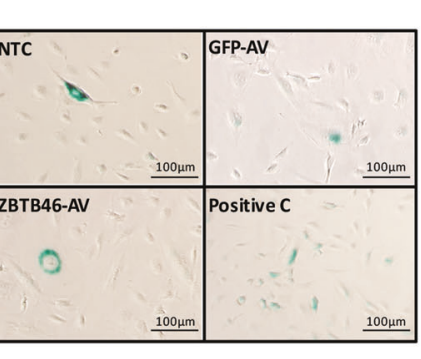

G

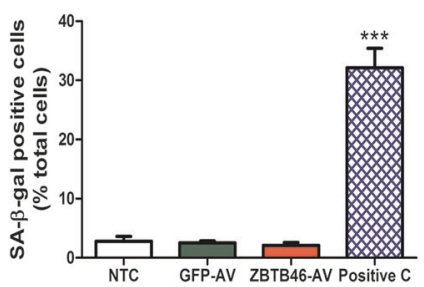

(early apoptotic cells) using flow cytometry (gated on live GFP + cells) showed no significant difference among groups. d Representative images of adenovirus-infected HAECs stained with Ki67 (red, marker of proliferation) and DAPI (blue, nuclei). e Quantification of Ki67positive cells as percentage of total GFP + cells using ImageJ software. $* * * P<0.001$, two-tailed $t$-test, $n=6-8 /$ group. f Representative images staining for SA- $\beta$-galactosidase in different groups of HAECs. HAECs were exposed to repeated $5 \%$ ethanol treatment to induce senescence as a positive control (Positive C) for the assay. g Quantification of SA- $\beta$-gal-positive cells ( $\%$ of total cells) in different groups of cells, manually counted and averaged from five field of views in each well. $* * * P<0.001$, compared to GFP control, one-way ANOVA, $n=4$

Importantly, downregulation of ZBTB46 using siRNA under LS conditions negated the inhibitory effect of LS on cell proliferation (Fig. 5a) showing an important role for ZBTB46 expression in LS-mediated inhibition of EC proliferation. On the other hand, overexpressing ZBTB46 using adenoviral transduction led to significant reduction of EC proliferation under OS, showing that in the OS proliferative condition ZBTB46 overexpression can also hinder EC proliferation (Fig. 5b).

\section{Full-length ZBTB46 locates to the nucleus, but a truncated version lacking the zinc finger domains remains mostly in the cytoplasm and does not significantly affect cell proliferation}

In order to elucidate the mechanism of ZBTB46 overexpression affecting cell proliferation, we first assessed its localization in the cell. Since we found no suitable ZBTB46 antibodies for immunostaining of human cells, we used two versions of FLAG-tagged ZBTB46-overexpressing vectors, one expressing the full-length, and a second truncated version lacking the zinc finger domains and the predicted 


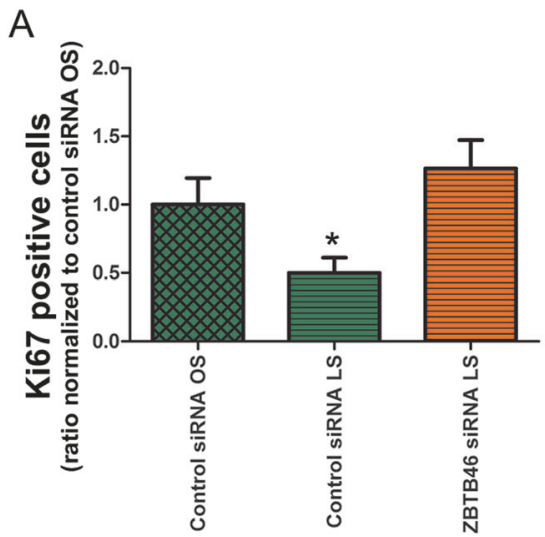

Fig. 5 ZBTB46 downregulation negates the inhibitory role of LS on EC proliferation, while ZBTB46 overexpression inhibits EC proliferation under OS condition. HAECs were cultured on collagen coated slide chambers and exposed to either LS $\left(15 \mathrm{dyn} / \mathrm{cm}^{2}\right)$ or OS $\left( \pm 5 \mathrm{dyn} / \mathrm{cm}^{2}\right)$ conditions for 2 days using the Ibidi shear stress system

nuclear localization site. Using immunofluorescence and anti-FLAG antibody, we found that the full-length FLAGtagged ZBTB46 located mostly to the nucleus; however, the FLAG-tagged truncated version of ZBTB46 did not translocate to the nucleus (Fig. 6a). As expected the FLAGtagged full-length ZBTB46 led to decreased cell proliferation compared to control; however, the truncated version of ZBTB46 had significantly less effect on cell proliferation (Fig. 6b), suggesting that nuclear localization is important in the effect of ZBTB46 on cell proliferation.

\section{ZBTB46 overexpression suppresses multiple cell cycle genes expression as well as Rb protein expression and phosphorylation}

To further explore the molecular basis of reduced cell proliferation in HAECs in response to ZBTB46 overexpression, cell cycle gene expression levels were compared in ZBTB46-transduced and control cells by qPCR. ZBTB46 overexpression led to a broad suppression of genes involved in cell cycle progression including cyclins (CCNs) CCNA2, CCNB1, CCND1, and CCNE2, as well as cyclindependent kinases (CDKs) CDK1, CDK2, CDK4, CDK6, and the retinoblastoma protein $(\mathrm{Rb} 1)$. On the other hand, ZBTB46 overexpression led to an increase in one of the CDK inhibitors, CDKN1A (p21), but no significant change in the CDK inhibitor CDKN1B (p27) as shown in Fig. 7a. The main endpoint of interaction between cyclins and CDKs is the phosphorylation of retinoblastoma protein $(\mathrm{Rb})$. We therefore assessed $\mathrm{Rb}$ protein phosphorylation by Western blot. Correspondingly, we observed a significant reduction in protein contents of total $\mathrm{Rb}$ and phosphorylated $\mathrm{Rb}$ protein (pRb) (Fig. 7b, c) as well as decreased ratio of $\mathrm{pRb}$ over total $\mathrm{Rb}$ levels (Fig. 7d). These results
$\mathrm{B}$

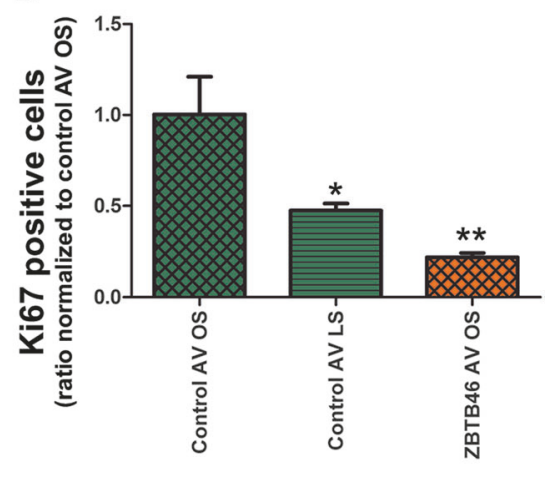

following treatment with a ZBTB46 siRNA or control siRNA, and b ZBTB46-AV or control-AV. Quantification of Ki67-positive cells as percentage of total EC was performed using ImageJ software, normalized to the control OS condition. $* P<0.05$, $* * P<0.01$, compared to control OS, $n=3-6 /$ group

demonstrate that ZBTB46 overexpression significantly affects the cell cycle by regulating a number of genes involved in cell cycle progression.

\section{ZBTB46 overexpression reduces tube formation in a Matrigel tube formation assay}

Angiogenesis is a process which is highly dependent on EC proliferation. In order to examine the comprehensive effect of ZBTB46 overexpression on the functionality of ECs in regard to angiogenesis, we used the Matrigel tube formation assay which is widely used as a surrogate for angiogenic capacity of ECs in vitro. ZBTB46 overexpressing HAECs formed significantly less tubules (Fig. 8a) when compared with control cells, with less total tube length and branching points (Fig. 8b, c). These results suggest a role for ZBTB46 in decreasing the angiogenic capacity of ECs.

\section{Discussion}

In the current study, we show for the first time that ZBTB46 is expressed in quiescent human ECs in vitro in addition to murine ECs in vivo, is regulated by shear stress and cell confluence, and that it can inhibit the proliferation of ECs in vitro. Furthermore, we show that ZBTB46 may exert its anti-proliferative effects through regulation of genes involved in the cell cycle including cyclins and CDKs, as well as the inhibitor of cyclin-dependent kinases CDKN1A $\left(\mathrm{p} 21^{\mathrm{Cip} 1}\right.$ ), culminating in reduced levels of phosphorylated retinoblastoma protein in ECs and increased number of cells in $\mathrm{G}_{0} / \mathrm{G}_{1}$ phase, leading to a functional change in ECs as seen with decreased tube formation in an in vitro model of angiogenesis. These results suggest a potential inhibitory 

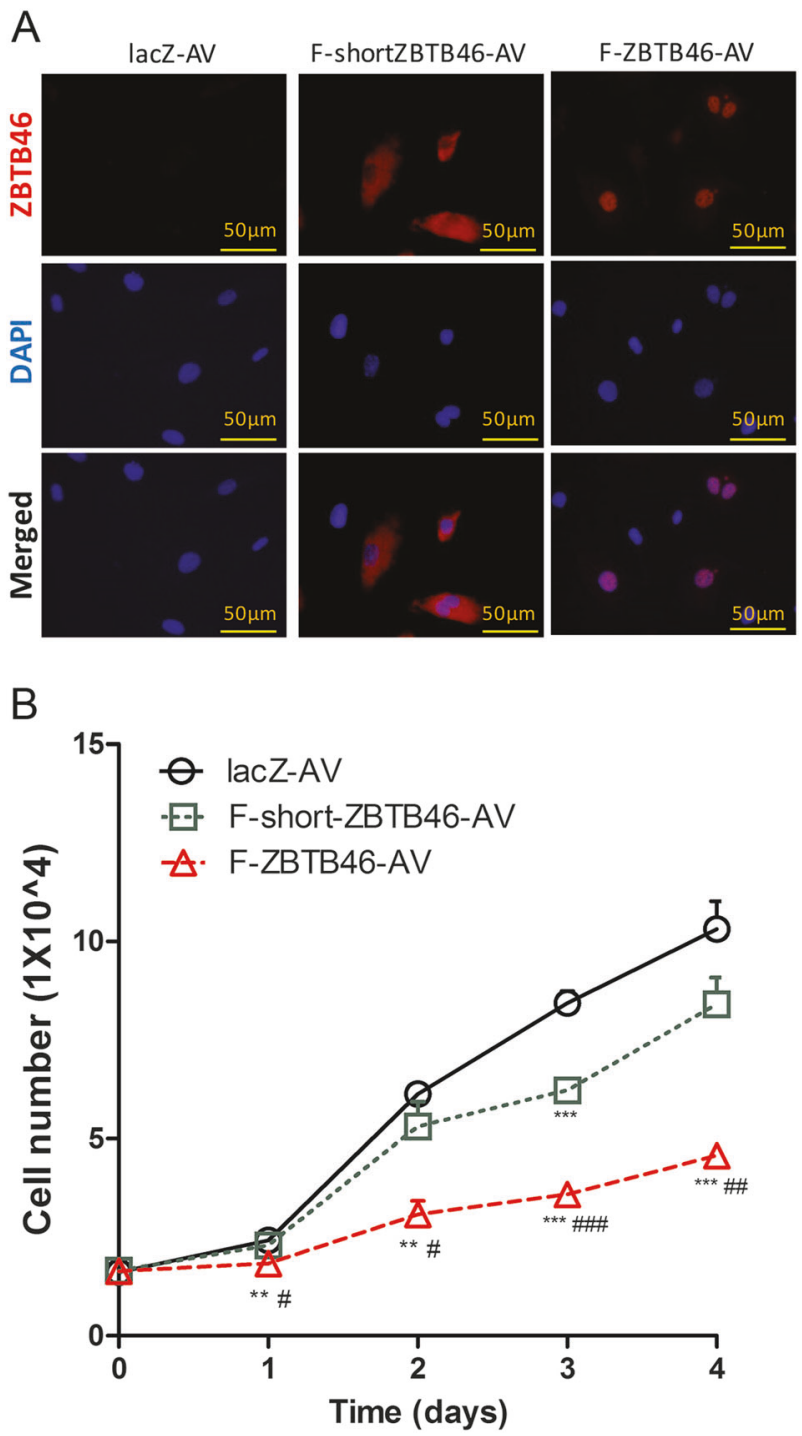

Fig. 6 Full-length but not truncated version of transduced ZBTB46 exhibits nuclear localization and suppresses cell proliferation in HAECs. a Representative images showing immunofluorescent staining of FLAG-tagged full-length ZBTB46 (F-ZBTB46-AV) or FLAGtagged truncated version ZBTB46 protein (F-shortZBRB46-AV) compared to control lacZ adenovirus-infected cells (lacZ-AV) using an anti-FLAG antibody (red), and DAPI nuclear counter stain (blue). b Adenovirus-infected cells were seeded at similar sub-confluent density in 24-well plates 1 day post infection. Cell numbers were counted manually every day for 4 days. $* P<0.05$, $* * P<0.01$, $* * * P<0.001$ compared to lacZ-AV, $\# P<0.05, \# \# P<0.01$, \#\#\#P<0.001 compared to F-shortZBTB46-AV, one-way ANOVA, $n=4$

role for ZBTB46 in various conditions where EC proliferation plays a significant role such as vascularization of cancerous tumors, pulmonary arterial hypertension, and atherosclerosis, while elucidating a novel mechanism through which altered flow and shear stress patterns may affect EC gene expression and proliferation.

ZBTB46 is a transcription factor belonging to the BTB/ POZ-ZF family of transcription repressors. While ZBTB46 expression was recently noted in ECs of the splenic vasculature [1], its role in ECs is unknown. ECs lining the arteries of adult humans are mostly present in a confluent monolayer exposed to unidirectional laminar shear stress, and are quiescent with a low turnover rate, but exhibit a higher turnover rate in areas exposed to disturbed flow [47]. Since other members of the BTBD/POZ-ZF family of transcription factors have been shown to act as a transcription repressor and affect cell proliferation [25], we hypothesized that ZBTB46 contributes to the quiescent state of ECs and its downregulation is required for EC proliferation. To test this hypothesis, we first examined whether ZBTB46 is differentially expressed in quiescent ECs vs. proliferating ECs in vivo. As disturbed blood flow (characterized by low and oscillatory shear stress) is a known stimulus for EC activation both in vivo and in vitro, we used an established method of inducing disturbed flow in a mouse model which also allows collection of EC-enriched RNA [18, 19], and found that quiescent ECs in the mouse common carotid artery express a baseline level of ZBTB46, which is significantly downregulated in activated ECs in the partially ligated common carotid artery exposed to disturbed flow (Fig. 1a). This was further confirmed using a second model comparing ECs chronically exposed to stable laminar shear and disturbed flow areas (Fig. 1b). Shear stress is known to affect ECs through multiple mechanisms, including regulation of gene expression, miRNA expression, and DNA methylation, modulated through integrins, primary cilia, adhesion molecules, gap junctions, ion channels, and more [26-35]. Further studies are needed to elucidate the mechanisms involved in shear stress regulation of ZBTB46 in ECs. As previously reported, arterial ECs in wild-type mice have a low proliferation rate (even in areas of disturbed flow), but baseline EC proliferation can be seen more prominently in ApoE KO mice, which is significantly increased in areas of disturbed flow [7]. We therefore performed the partial carotid ligation procedure in ApoE KO mice and found that similar to what is previously reported, EC proliferation is increased in response to disturbed flow in the partially ligated carotid (Supplemental Fig. 2). In the current study we did not focus on the origin of these proliferating cells, and therefore cannot determine if these proliferating cells represent a stem cell repair sub population as previously asserted [7], or the fully differentiated existing arterial EC which have now become more proliferative. However, when we assessed the gene expression level of the CDK inhibitor CDKN1A (p21 ${ }^{\text {Cip1 }}$, which has been reported to be shear sensitive and inhibit EC proliferation) [16] in wild-type C57B1/6 mice in vivo, we found that CDKN1A mRNA levels were significantly reduced in the partially ligated artery (Fig. 1c). Overall, these results show a correlation between downregulation of ZBTB46 and CDKN1A by disturbed flow in vivo, and suggest they may 


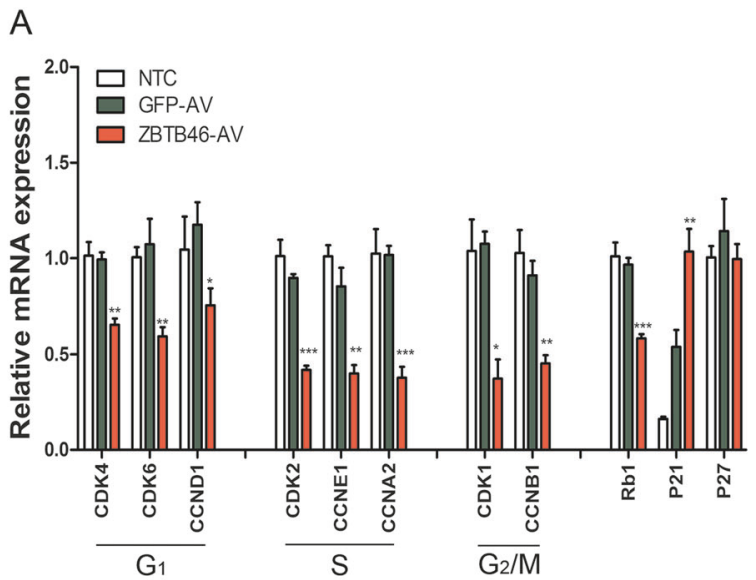

Fig. 7 ZBTB46 overexpression results in a broad suppression of multiple cell cycle genes and correspondingly a reduction in phosphorylated retinoblastoma ( $\mathrm{pRb}$ ) protein content in HAECs. a Relative mRNA expression of various genes involved in cell cycle regulation in HAECs overexpressing ZBTB46 compared to controls by qPCR, grouped by their predominant known roles in various phases of the cell

contribute to the increased EC proliferation seen in areas of disturbed flow in vivo under conditions that stimulate EC proliferation.

The effect of shear stress on regulating ZBTB46 in ECs was further confirmed in vitro using an established coneand-plate model (Fig. 2d-f). It should be noted that many effects of shear stress on EC gene regulation are affected by the magnitude of the shear stress as well as the shear rate, pulsatility, and the time frame of shear exposure. The effect of these shear stress parameters on ZBTB46 expression need to be further studied. However, the magnitude of changes seen in ZBTB46 expression under OS vs. LS conditions used in vitro in this study roughly correlate with the magnitude of in vivo changes observed, suggesting that the parameters used in vitro are an acceptable starting point to study the effects of shear stress on ZBTB46 expression. When isolated and cultured in vitro under static conditions in presence of required nutrients and growth factors, ECs become proliferative and eventually produce a confluent monolayer at which point they exhibit contact inhibition. Notably, we also found that ECs proliferating in regular culture conditions in vitro express very low levels of ZBTB46 which increases as the cells become confluent (Fig. 2a-c). Higher levels of ZBTB46 expression in contact inhibited cells (post-confluence) as well as cells exposed to steady laminar shear suggests a potential role for ZBTB46 in inhibiting EC proliferation. We therefore focused our studies to examine whether ZBTB46 expression led to decreased EC proliferation. As proliferating ECs express minimal levels of ZBTB46, a further knockdown of ZBTB46 in cells cultured under static conditions using siRNA would not be useful in assessing its role on cell
B

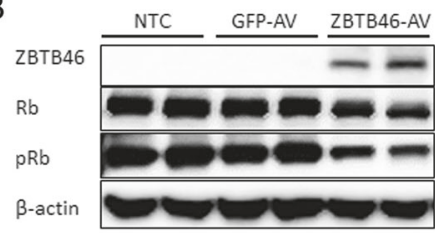

C
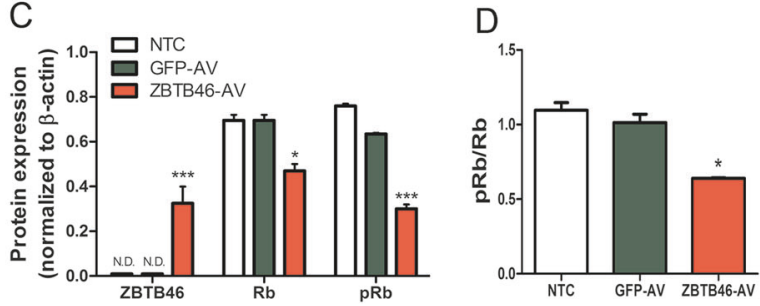

cycle. b Representative Western blots showing reduced total $\mathrm{Rb}$ and $\mathrm{pRb}$ protein content in ZBTB46 transducing HAECs compared to control cells. $\mathbf{c}$ Quantification of $\mathrm{Rb}$ and $\mathrm{pRb}$ protein content based on Western blots. $\mathbf{d}$ Calculated ratio of $\mathrm{pRb}$ to total $\mathrm{Rb}$ showed decreased $\mathrm{Rb}$ protein phosphorylation. $* P<0.05$, $* * * P<0.001$ compared to GFP-AV, one-way ANOVA, $n=4-6 /$ group

proliferation and we proceeded with an overexpressing strategy using adenoviral-mediated overexpression of ZBTB46 in HAECs, which led to a robust expression of ZBTB46 (Fig. 3a-c). Using this approach, we found that cells overexpressing ZBTB46 showed significant decrease in proliferation throughout a 4-day experimental timeframe (Fig. 3d), showing that ZBTB46 has a significant role on inhibiting EC proliferation. Given the observed reduction in EC proliferation in response to ZBTB46 overexpression and the observed correlation between gene expression levels of ZBTB46 and CDKN1A in areas of disturbed flow, we studied the effect of ZBTB46 overexpression on cell cycle using flow cytometry. Cells overexpressing ZBTB46 showed a significant increased frequency of cells in the $G_{0} / G_{1}$ phase and a decreased frequency of cells in the $S$ phase, with no significant effect on number of cells undergoing apoptosis (Fig. 4a-c). To distinguish nonproliferating cells in $\mathrm{G}_{0}$ from cells in the $\mathrm{G}_{1}$ phase of the cell cycle, we performed immunofluorescent staining with Ki67 antibody in ZBTB46-overexpressing and control cells. Ki67 protein is present during all active phases of the cell cycle $\left(G_{1}, S, G_{2}\right.$, and $\left.M\right)$, but is absent from resting cells $\left(G_{0}\right)$ and has been widely used as a marker of proliferating cells [24]. We found that cells overexpressing ZBTB46 showed significantly less frequency of Ki67-positive cells (Fig. 4d, e), suggesting that ZBTB46 overexpression led to cells exiting the cell cycle. Senescent cells are also non-proliferative (Ki67-negative), and eventually undergo apoptosis. To assess if ZBTB46 overexpression was leading to cell senescence, we performed a SA- $\beta$-gal staining assay for senescent cells, which revealed no increase in number of positively stained cells in the ZBTB46-overexpressing cells 
A
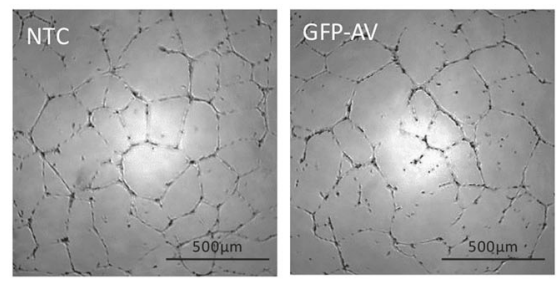

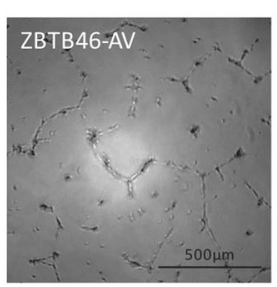

Fig. 8 ZBTB46 overexpression leads to reduced tube formation and branch points in a Matrigel tube formation assay. a Representative images of HAECs cultured on Matrigel with or without ZBTB46

(Fig. 4f, g). The lack of increased SA- $\beta$-gal-positive staining in ZBTB46-overexpressing cells, along with no indication of increased apoptotic cells, showed that ZBTB46 overexpression did not induce cell senescence, and therefore the increased number of $G_{0}$ cells can be attributed to cell quiescence rather than senescence. We then examined if ZBTB46 affects the changes in EC proliferation observed in different shear stress treatment of ECs. Our results showed that as expected, exposure to LS condition inhibited EC proliferation compared to OS, but this inhibitory effect of LS on EC proliferation was rescued when ZBTB46 was downregulated by siRNA (Fig. 5a). On the other hand, adenoviral overexpression of ZBTB46 reduced EC proliferation under OS conditions (Fig. 5b). These results show that ZBTB46 plays an important role in the shear stress regulation of EC proliferation.

Meredith et al. have shown that ZBTB46 affects gene expression in cDCs and a ChIP-Seq assay identified a large number of cell cycle genes as potential ZBTB46 targets in cDCs [3]. Having found the effect of ZBTB46 on EC proliferation, we next assessed the effect of ZBTB46 overexpression on the expression of genes involved in cell cycle. We first questioned whether ZBTB46 indeed localizes to the nucleus in ECs in order to affect gene expression. Currently, no suitable anti-ZBTB46 antibodies are available for performing immunofluorescence studies in human cells, therefore we used a FLAG-tagged version of ZBTB46-expressing vector to overexpress FLAG-tagged ZBTB46 in ECs and used anti-FLAG antibody to locate the protein in ECs using immunofluorescence. Our results showed that ZBTB46 did indeed localize predominantly to the nucleus; however, a truncated version of ZBTB46 lacking the zinc finger DNA binding domains as well as the predicted nuclear localization site was found predominantly in the cytoplasm (Fig. 6a). We also confirmed that the FLAG-tagged ZBTB46 overexpression led to decreased cell proliferation, but found the truncated version of ZBTB46 had significantly less effect on cell proliferation overall, showing a significant difference with the control cell population only at day 3 which requires further investigation (Fig. 6b). We then proceeded to assess the effect of
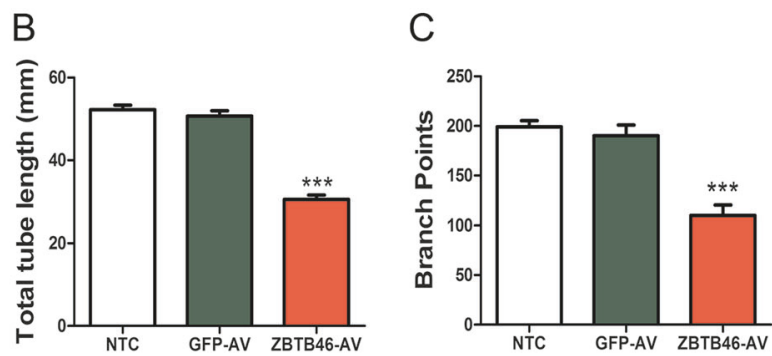

overexpression. b, c Quantification of total tube length and branch points using ImageJ software. $* * * P<0.001$ compared to GFP-AV, one-way ANOVA, $n=4-6 /$ group

ZBTB46 overexpression on cell cycle genes including the cyclins, CDKs, CDK inhibitors, and Rb1. Interestingly, ZBTB46 overexpression had a widespread effect, downregulating gene expression of all the cyclins and CDKs tested, with the most significant effect on cyclin A2 (CCNA2) and CDK2, both known to be involved in progression of the cell cycle through the $\mathrm{S}$ phase of the cell cycle. At the same time, the gene expression of the CDK inhibitor CDKN1A (p21) showed a significant increase in the ZBTB46-overexpressing cells, but no significant change was observed for CDKN1B (p27) (Fig. 7a). Rb1 gene expression as well as $\mathrm{Rb}$ protein level and phosphorylation ratio were also reduced in ZBTB46-overexpressing cells (Fig. 7b-d). Further research is needed to assess whether the gene expression effects of ZBTB46 overexpression on cell cycle is a direct effect due to the binding of ZBTB46 to the promoter sites of these genes or a secondary effect. Interestingly, laminar shear stress has been shown to inhibit EC proliferation in vitro via inducing CDKN1A (but not CDKN1B) and decreased Rb phosphorylation in ECs [15, 16]. While we found that ZBTB46 overexpression in ECs increased CDKN1A gene expression, it remains to be seen if shear stress induction of CDKN1A in ECs is mediated through ZBTB46. Prior ChIP-Seq data in cDCs showed both CDKN1A and CDKN1B as potential targets of ZBTB46 (found in list of genes in the supplementary file) [3], suggesting that ZBTB46 may indeed directly affect the gene expression of these CDK inhibitors. Other important transcription factors affecting EC proliferation such as NFkB, AP1, EGR1, KLF2, and KLF4 are also regulated by shear stress and whether ZBTB46 cooperates with these and other transcription factors to mediate the effects of shear stress remains to be seen [8-14]. The above ChIP-Seq data in cDCs also listed KLF2, KLF4, various subunits of AP1, and NFKB2 as targets of ZBTB46; however, the potential interactions between these transcription factors have not been the focus of the current study and require further investigation. Future studies will focus on the effect of ZBTB46 on EC gene expression under various shear stress conditions including its effect on CDKN1A expression as well as other shear responsive genes and its interaction with 
other transcription factors. Finally, to assess the overall effect of ZBTB46 overexpression in a functional in vitro assay, we used a Matrigel tube formation assay and found that ZBTB46 overexpression leads to decreased tube formation (Fig. 8), suggestive of decreased angiogenic capacity of ECs overexpressing ZBTB46.

In summary, we have identified a novel flow-sensitive transcription factor which inhibits EC proliferation and may thus contribute to the underlying mechanisms involved in multiple diseases. EC proliferation plays an important role in many disease processes such as atherosclerosis, pulmonary hypertension, postsurgical neointimal hyperplasia, in-stent restenosis, vein bypass graft failure, and transplant vasculopathy. Importantly, atherosclerotic lesions have a propensity to occur in areas of disturbed flow and we have previously shown that disturbed flow contributes to accelerated atherosclerosis in an animal model [18]. More recently, ECs exposed to disturbed flow have been shown to contribute to neointimal proliferation following drug-eluting bioresorbable vascular scaffold implantation [36]. ZBTB46 may therefore play a role in disturbed flow-mediated atherosclerosis and in-stent restenosis. Further research is required to investigate the potential role of ZBTB46 in specific pathologic conditions and whether it can be targeted in a therapeutic manner.

Acknowledgements This work was supported by funding from National Institutes of Health Grant HL124292 to AR and HL095070 to HJ.

\section{Compliance with ethical standards}

Conflict of interest The authors declare that they have no conflict of interest.

Open Access This article is licensed under a Creative Commons Attribution-NonCommercial-NoDerivatives 4.0 International License, which permits any non-commercial use, sharing, distribution and reproduction in any medium or format, as long as you give appropriate credit to the original author(s) and the source, and provide a link to the Creative Commons license. You do not have permission under this license to share adapted material derived from this article or parts of it. The images or other third party material in this article are included in the article's Creative Commons license, unless indicated otherwise in a credit line to the material. If material is not included in the article's Creative Commons license and your intended use is not permitted by statutory regulation or exceeds the permitted use, you will need to obtain permission directly from the copyright holder. To view a copy of this license, visit http://creativecommons.org/licenses/by-nc-nd/4.0/.

\section{References}

1. Satpathy AT, Kc W, Albring JC, et al. Zbtb46 expression distinguishes classical dendritic cells and their committed progenitors from other immune lineages. J Exp Med. 2012;209:1135-52.

2. Meredith MM, Liu K, Darrasse-Jeze G, et al. Expression of the zinc finger transcription factor zDC (Zbtb46, Btbd4) defines the classical dendritic cell lineage. J Exp Med. 2012;209:1153-65.
3. Meredith MM, Liu K, Kamphorst AO, et al. Zinc finger transcription factor $\mathrm{zDC}$ is a negative regulator required to prevent activation of classical dendritic cells in the steady state. J Exp Med. 2012;209:1583-93.

4. Mahmoud MM, Kim HR, Xing R, et al. TWIST1 integrates endothelial responses to flow in vascular dysfunction and atherosclerosis. Circ Res. 2016;119:450-62.

5. Schober A, Nazari-Jahantigh M, Wei Y, et al. MicroRNA-126-5p promotes endothelial proliferation and limits atherosclerosis by suppressing Dlk1. Nat Med. 2014;20:368-76.

6. Suo J, Ferrara DE, Sorescu D, et al. Hemodynamic shear stresses in mouse aortas: implications for atherogenesis. Arterioscler Thromb Vasc Biol. 2007;27:346-51.

7. Foteinos G, Hu Y, Xiao Q, et al. Rapid endothelial turnover in atherosclerosis-prone areas coincides with stem cell repair in apolipoprotein E-deficient mice. Circulation. 2008;117:1856-63.

8. Clark PR, Jensen TJ, Kluger MS, et al. MEK5 is activated by shear stress, activates ERK5 and induces KLF4 to modulate TNF responses in human dermal microvascular endothelial cells. Microcirculation. 2011;18:102-17.

9. Villarreal G Jr., Zhang Y, Larman HB, et al. Defining the regulation of KLF4 expression and its downstream transcriptional targets in vascular endothelial cells. Biochem Biophys Res Commun. 2010;39:984-9.

10. Hamik A, Lin Z, Kumar A, et al. Kruppel-like factor 4 regulates endothelial inflammation. J Biol Chem. 2007;282:13769-79.

11. SenBanerjee $S$, Lin Z, Atkins GB, et al. KLF2 Is a novel transcriptional regulator of endothelial proinflammatory activation. $\mathrm{J}$ Exp Med. 2004;199:1305-15.

12. Schwachtgen JL, Houston P, Campbell C, et al. Fluid shear stress activation of egr-1 transcription in cultured human endothelial and epithelial cells is mediated via the extracellular signal-related kinase 1/2 mitogen-activated protein kinase pathway. J Clin Invest. 1998;101:2540-9.

13. Lan Q, Mercurius KO, Davies PF. Stimulation of transcription factors NF kappa B and AP1 in endothelial cells subjected to shear stress. Biochem Biophys Res Commun. 1994;201:950-6.

14. Abdel-Malak NA, Mofarrahi M, Mayaki D, et al. Early growth response-1 regulates angiopoietin-1-induced endothelial cell proliferation, migration, and differentiation. Arterioscler Thromb Vasc Biol. 2009;29:209-16.

15. Lin K, Hsu PP, Chen BP, et al. Molecular mechanism of endothelial growth arrest by laminar shear stress. Proc Natl Acad Sci USA. 2000;97:9385-9.

16. Akimoto S, Mitsumata M, Sasaguri T, et al. Laminar shear stress inhibits vascular endothelial cell proliferation by inducing cyclindependent kinase inhibitorp21(Sdi1/Cip1/Waf1). Circ Res. 2000;86:185-90.

17. Chiu JJ, Chien S. Effects of disturbed flow on vascular endothelium: pathophysiological basis and clinical perspectives. Physiol Rev. 2011;91:327-87.

18. Nam D, Ni CW, Rezvan A, et al. Partial carotid ligation is a model of acutely induced disturbed flow, leading to rapid endothelial dysfunction and atherosclerosis. Am J Physiol Heart Circ Physiol. 2009;297:H1535-43.

19. Nam D, Ni CW, Rezvan A, et al. A model of disturbed flowinduced atherosclerosis in mouse carotid artery by partial ligation and a simple method of RNA isolation from carotid endothelium. J Vis Exp. 2010; 40:e1861.

20. Son DJ, Kumar S, Takabe W, et al. The atypical mechanosensitive microRNA-712 derived from pre-ribosomal RNA induces endothelial inflammation and atherosclerosis. Nat Commun. 2013;4:3000.

21. Sorescu GP, Sykes M, Weiss D, et al. Bone morphogenic protein 4 produced in endothelial cells by oscillatory shear stress stimulates an inflammatory response. J Biol Chem. 2003;278: 31128-35. 
22. Starke RD, Ferraro F, Paschalaki KE, et al. Endothelial von Willebrand factor regulates angiogenesis. Blood. 2011;117: 1071-80.

23. Li J, Cubbon RM, Wilson LA, et al. Orail and CRAC channel dependence of VEGF-activated $\mathrm{Ca} 2+$ entry and endothelial tube formation. Circ Res. 2011;108:1190-8.

24. Scholzen T, Gerdes J. The Ki-67 protein: from the known and the unknown. J Cell Physiol. 2000;182:311-22.

25. Siggs OM, Beutler B. The BTB-ZF transcription factors. Cell Cycle. 2012;11:3358-69.

26. Ni CW, Qiu H, Rezvan A, et al. Discovery of novel mechanosensitive genes in vivo using mouse carotid artery endothelium exposed to disturbed flow. Blood. 2010;116:e66-73.

27. Lee DY, Lee CI, Lin TE, et al. Role of histone deacetylases in transcription factor regulation and cell cycle modulation in endothelial cells in response to disturbed flow. Proc Natl Acad Sci USA. 2012;109:1967-72.

28. Kumar S, Kim CW, Simmons RD, et al. Role of flow-sensitive microRNAs in endothelial dysfunction and atherosclerosis: mechanosensitive athero-miRs. Arterioscler Thromb Vasc Biol. 2014;34:2206-16.

29. Dunn J, Qiu H, Kim S, et al. Flow-dependent epigenetic DNA methylation regulates endothelial gene expression and atherosclerosis. J Clin Invest. 2014;124:3187-99.
30. Tzima E, Irani-Tehrani M, Kiosses WB, et al. A mechanosensory complex that mediates the endothelial cell response to fluid shear stress. Nature. 2005;437:426-31.

31. Qin X, Wang X, Wang Y, et al. MicroRNA-19a mediates the suppressive effect of laminar flow on cyclin D1 expression in human umbilical vein endothelial cells. Proc Natl Acad Sci USA. 2010;107:3240-4.

32. Wang KC, Garmire LX, Young A, et al. Role of microRNA-23b in flow-regulation of $\mathrm{Rb}$ phosphorylation and endothelial cell growth. Proc Natl Acad Sci USA. 2010;107:3234-9.

33. Kumar S, Kim CW, Son DJ, et al. Flow-dependent regulation of genome-wide mRNA and microRNA expression in endothelial cells in vivo. Sci Data. 2014;1:140039.

34. Kwak BR, Back M, Bochaton-Piallat ML, et al. Biomechanical factors in atherosclerosis: mechanisms and clinical implications. Eur Heart J. 2014;35:3013-20, 3020a-3020d.

35. Traub O, Berk BC. Laminar shear stress: mechanisms by which endothelial cells transduce an atheroprotective force. Arterioscler Thromb Vasc Biol. 1998;18:677-85.

36. Bourantas CV, Papafaklis MI, Kotsia A, et al. Effect of the endothelial shear stress patterns on neointimal proliferation following drug-eluting bioresorbable vascular scaffold implantation: an optical coherence tomography study. JACC Cardiovasc Interv. $2014 ; 7: 315-24$. 